\title{
Hierarchical Plasmon Resonances in Fractal Structures
}

\author{
Isobel C. Bicket, ${ }^{* \dagger}{ }^{\dagger}$ Edson P. Bellido, ${ }^{\dagger}$ Danielle M. McRae, ${ }^{\ddagger}$ François \\ Lagugné-Labarthet, ${ }^{*, \ddagger}$ and Gianluigi A. Botton ${ }^{*, \dagger, \uparrow}$ \\ $\dagger$ McMaster University, 1280 Main Street West, Hamilton, ON, Canada, L8S 4 L8 \\ $\ddagger$ The University of Western Ontario, 1151 Richmond Street, London, ON, Canada, N6A \\ $5 B 7$ \\ \Canadian Light Source, 44 Innovation Boulevard, Saskatoon, SK, Canada, S7N 2 V3 \\ E-mail: bicketic@mcmaster.ca; flagugne@uwo.ca; gbotton@mcmaster.ca
}




\section{Hybridization of Edge and Cavity Modes}

Figure S1 displays experimental EELS maps of the higher order edge and cavity modes of the G0, G0.5, G1, G1.5, and G2 fractals. Hybridization of the dipolar modes of parent generations is shown in the main text. The edge modes of the G0 fractal hybridize when G0 is used to construct further fractal generations, as do the cavity modes.

Not only do the dipolar modes of G0 hybridize, as discussed in the main text, but the higher order G0 edge and cavity modes can also hybridize when higher fractal generations are built. When apertures are added to the G0 structure to create G1 and higher, the mode behaviour becomes very complex ${ }^{1}$ and the distinction between edge and cavity modes becomes blurred. For instance, when an aperture is added to G0, the lowest energy G0 cavity mode changes and behaves like an edge mode around the central G1 aperture. Concerning the additive fractals, in which the G0 parent is the same size throughout the construction of further generations, the cavity mode hybridizes to form multiple cavity modes with small energy differences. For example, as discussed in the main text, in G0.5, there are two G0 parent units and the lowest energy G0 cavity mode hybridizes to form symmetric and antisymmetric modes. In the G1 fractal, there are three G0 parents and a three-way hybridization of the lowest energy G0 cavity mode. Each successive generation increases the number

of modes seen in this spectral region. The natural plasmon linewidth, instrumental peak broadening, and the close energy spacing of so many hybridized cavity modes means that the spectral features above $1.5 \mathrm{eV}$ are all blurred into each other and no clear peaks can be distinguished as the fractal generation advances. Cavity modes are also known to have relatively weak EELS signal compared to other modes. ${ }^{2,3}$

The cavity modes are excited when the electron beam passes through the faces of the G0 parent prisms. Unfortunately, the scattering of the electron beam is strongest when it passes through the nanostructure, degrading the signal-to-noise ratio (SNR) for the cavity modes. Each of the spectra in Figure 2(b) (repeated in Figure S1(b) for ease of comparison) has been normalized to the zero loss peak, emphasizing the noise in the cavity mode spectra relative 
to spectra integrated over regions outside the nanostructures. The low SNR also explains the comparatively noisy EELS intensity maps of the cavity modes in Figure S1(a). A similar argument can be made for some of the higher order edge modes (e.g. peaks 8, 9, 10 of G1.5 in Figure S1(b)(iv)), which are integrated over very small areas and so have a poorer SNR than a larger integration area would provide. With the higher order fractal generations, the sheer number of modes present in the spectrum makes identifying individual peaks at higher energies very difficult, even with the local excitation provided by EELS spectrum imaging techniques. 
(a)
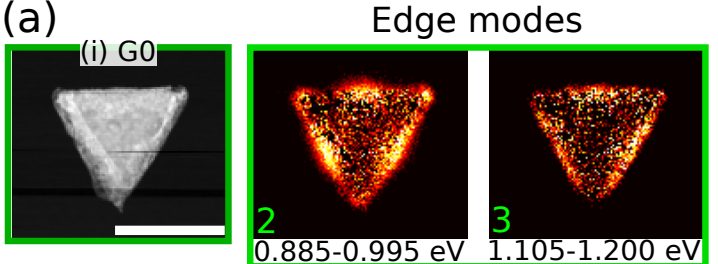

(ii) G0.5

$0.885-0.995 \mathrm{eV} 1.105-1.200 \mathrm{eV}$

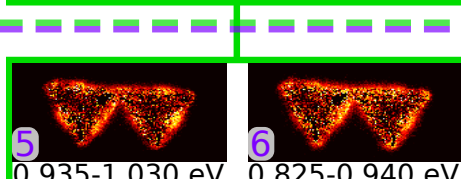

$0.935-1.030 \mathrm{eV}$

$0.825-0.940 \mathrm{eV}$

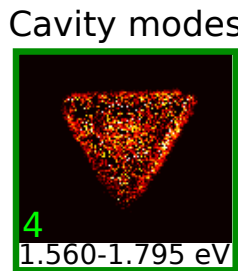

$1.560-1.795 \mathrm{eV}$ (b)

Wavelength $(\mathrm{nm})$

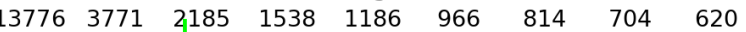

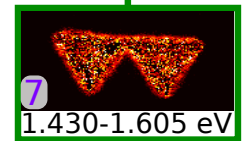

$1.430-1.605 \mathrm{eV}$
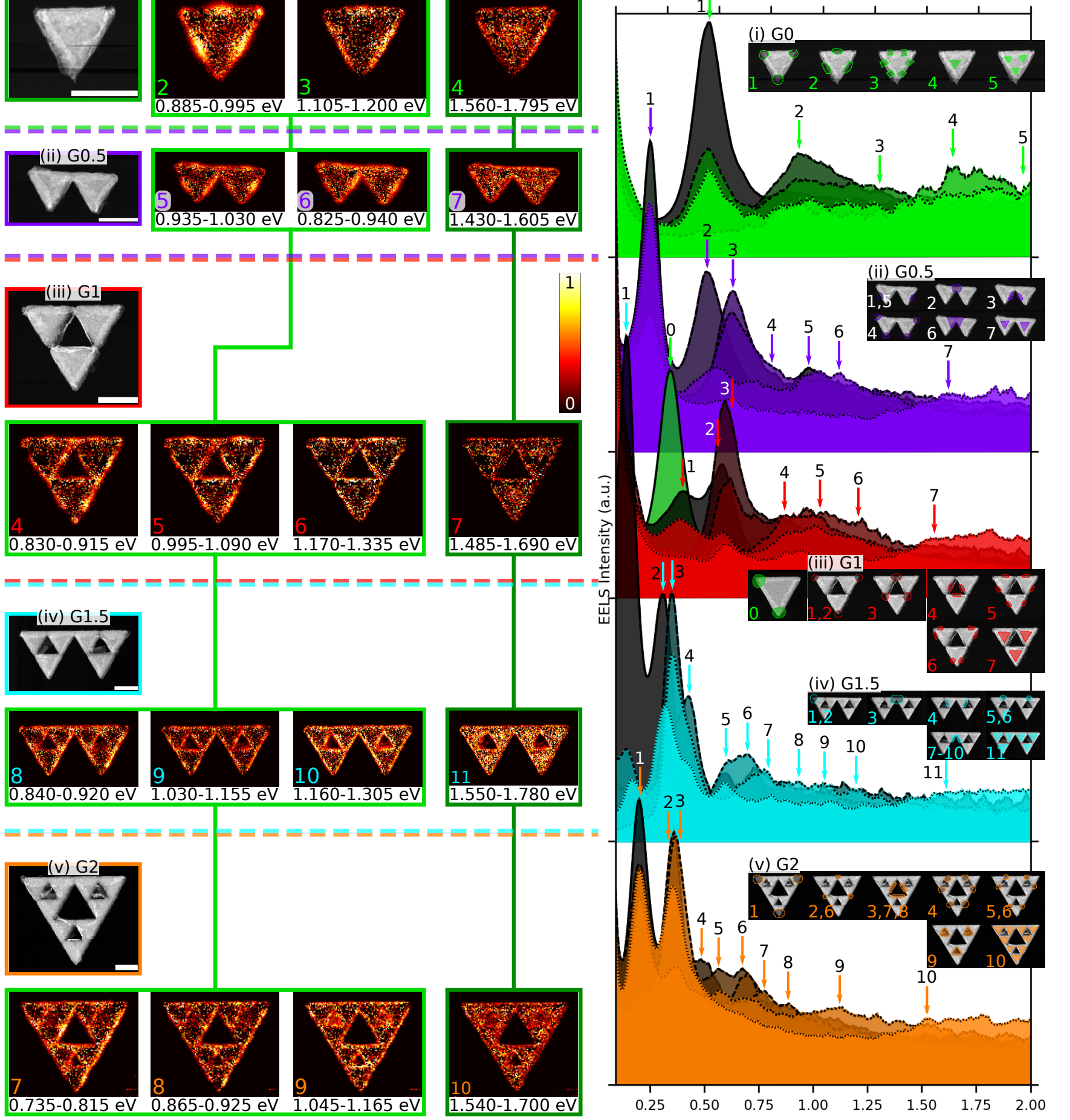

드는

Figure S1: Experimental EELS data for the edge and cavity modes of the fractal generations presented in the main text. (a) HAADF and EELS intensity maps for edge and cavity modes; the scalebar for the HAADF images is $500 \mathrm{~nm}$; each map is individually normalized and coloured according to the colour scale on the right. (b) EELS spectra repeated from Figure 2 for clarity and ease of comparison with the intensity maps. 


\section{FDTD Electric Field Calculations of G2}

Complementary to the surface charge maps presented in Figure 4 in the main text, Figure S2 - S9 show electric field calculations for eight modes on the generation 2 (G2) Sierpiński fractal structure. For all plots, polarization of the excitation light is given by the green arrow. Streamlines indicate $(x, y)$ field components and colours indicate the $z$ component of the field for each plot.

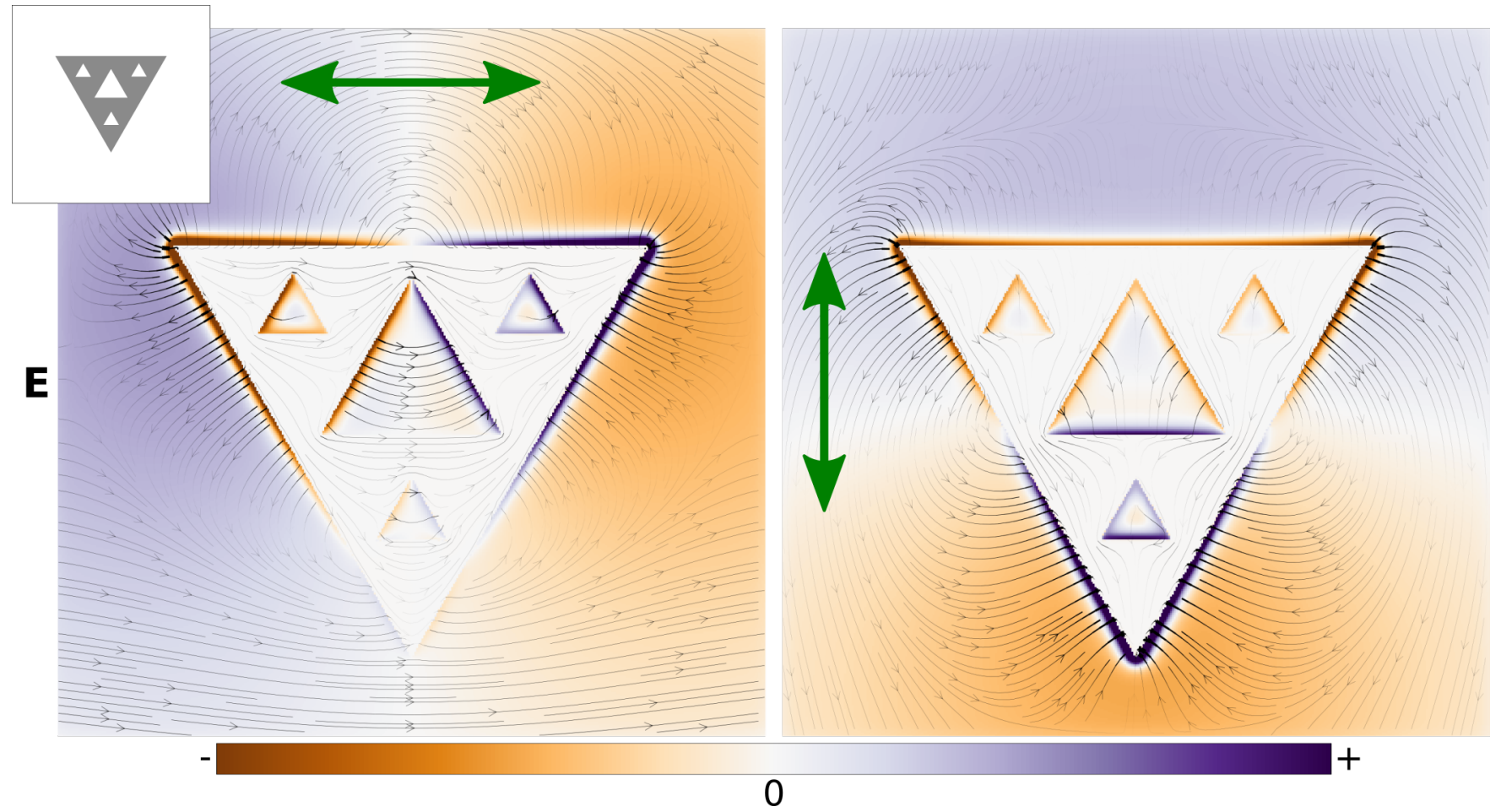

Figure S2: Simulated electric (E) fields for a plane just above the surface of the metal for a G2 fractal at $0.260 \mathrm{eV}(4772 \mathrm{~nm})$. 


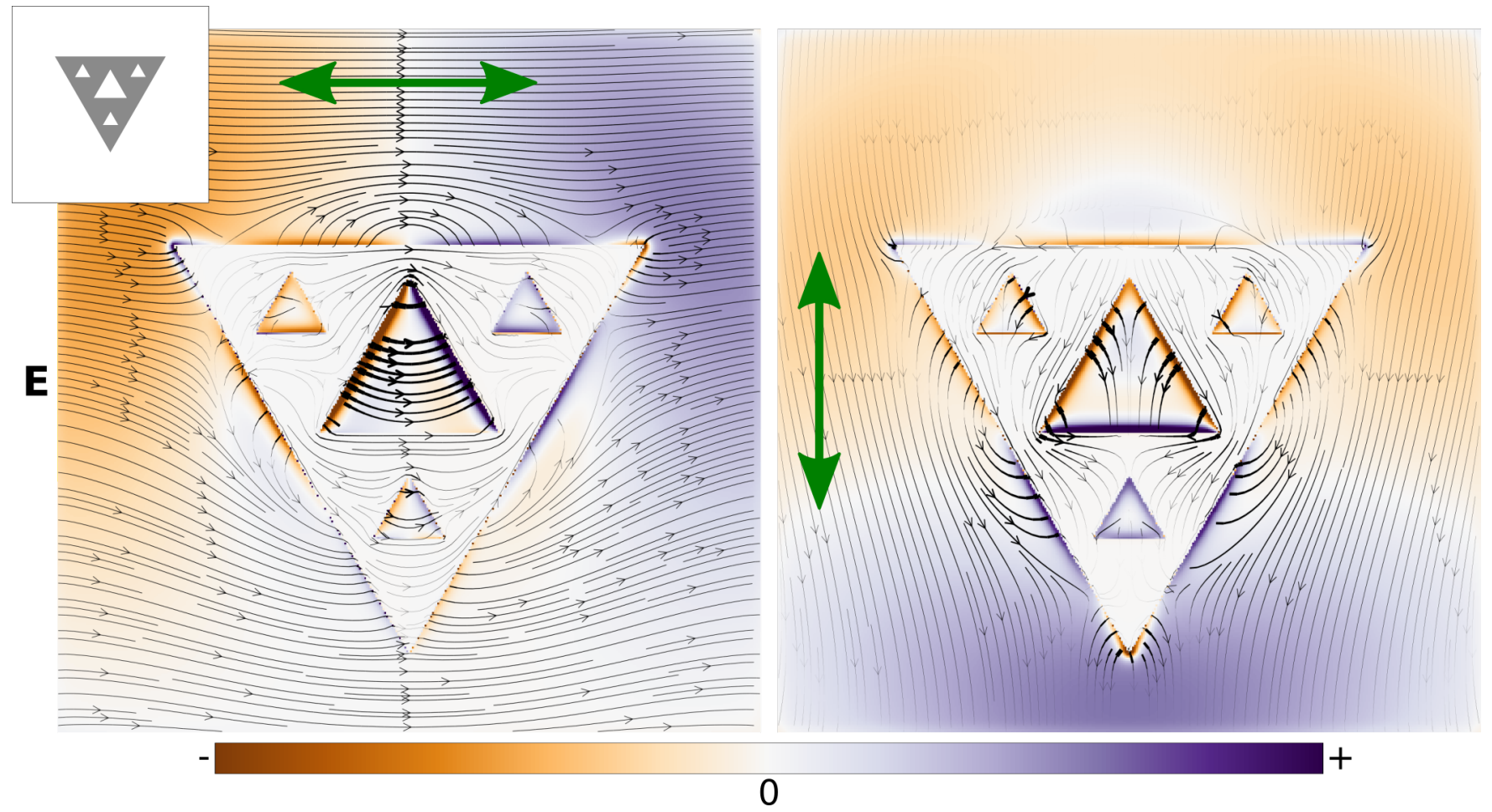

Figure S3: Simulated electric (E) fields for a plane just above the surface of the metal for a $\mathrm{G} 2$ fractal at $0.487 \mathrm{eV}(2545 \mathrm{~nm})$.

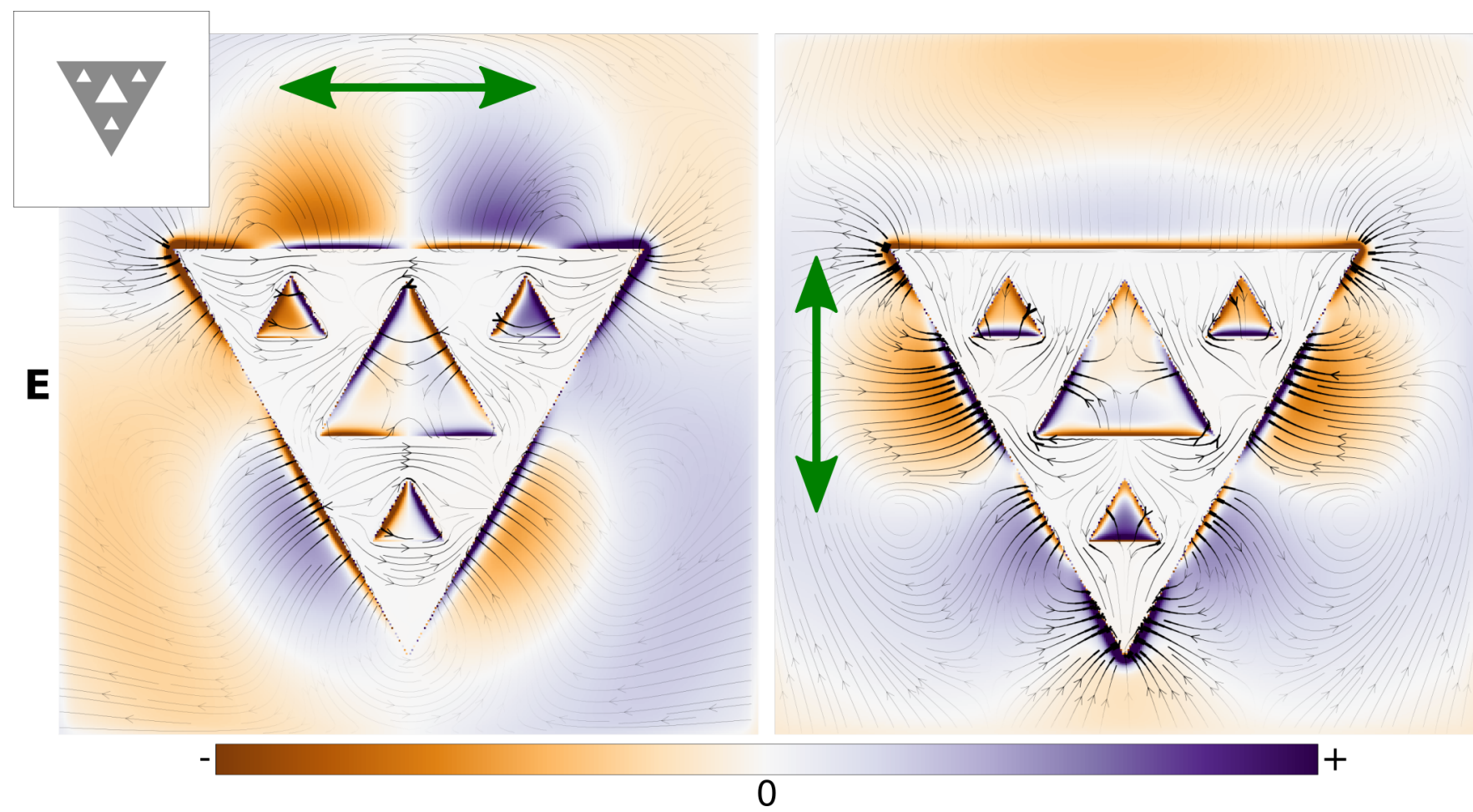

Figure S4: Simulated electric (E) fields for a plane just above the surface of the metal for a $\mathrm{G} 2$ fractal at $0.666 \mathrm{eV}(1863 \mathrm{~nm})$. 

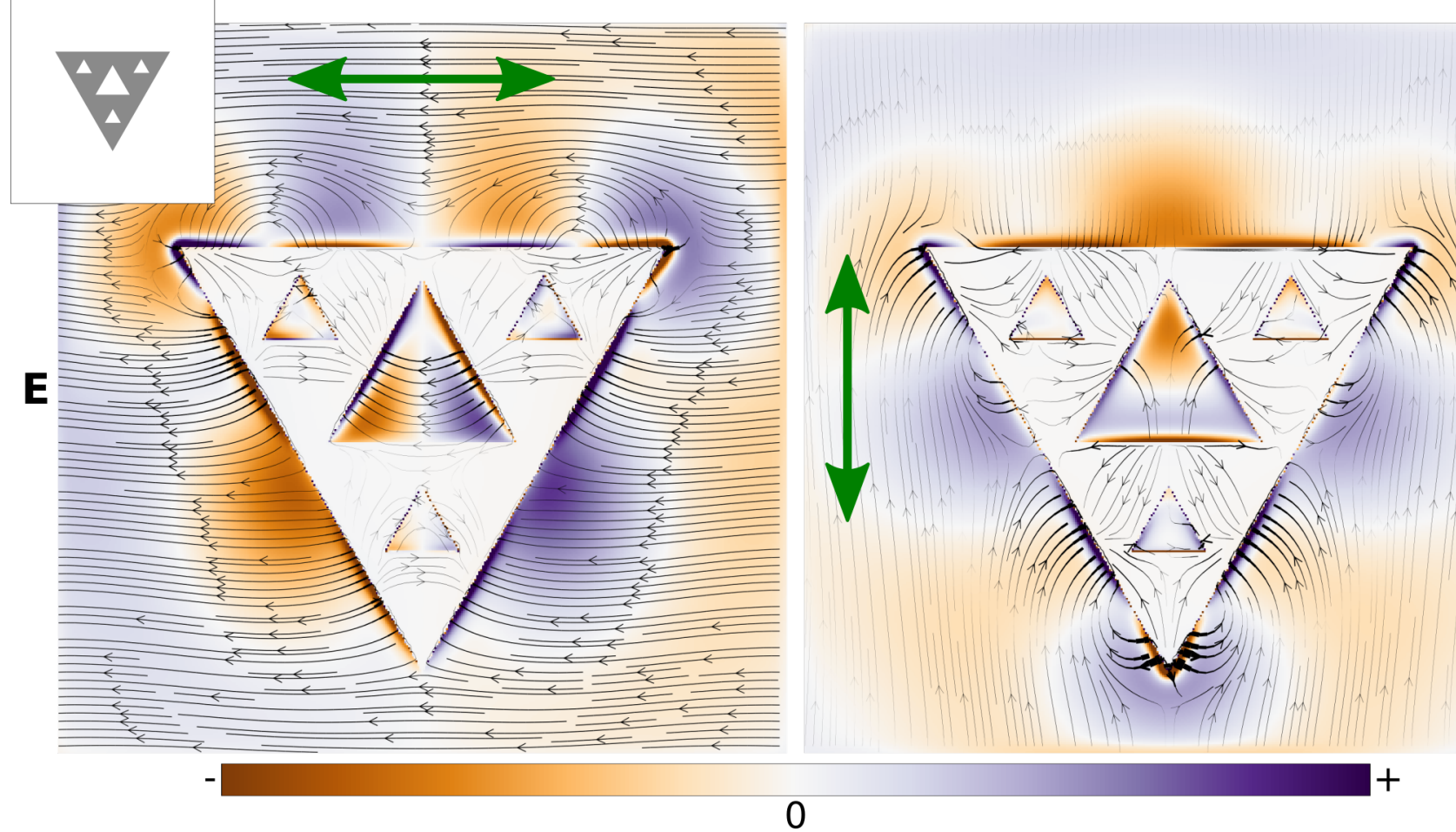

Figure S5: Simulated electric (E) fields for a plane just above the surface of the metal for a G2 fractal at $0.880 \mathrm{eV}(1409 \mathrm{~nm})$.

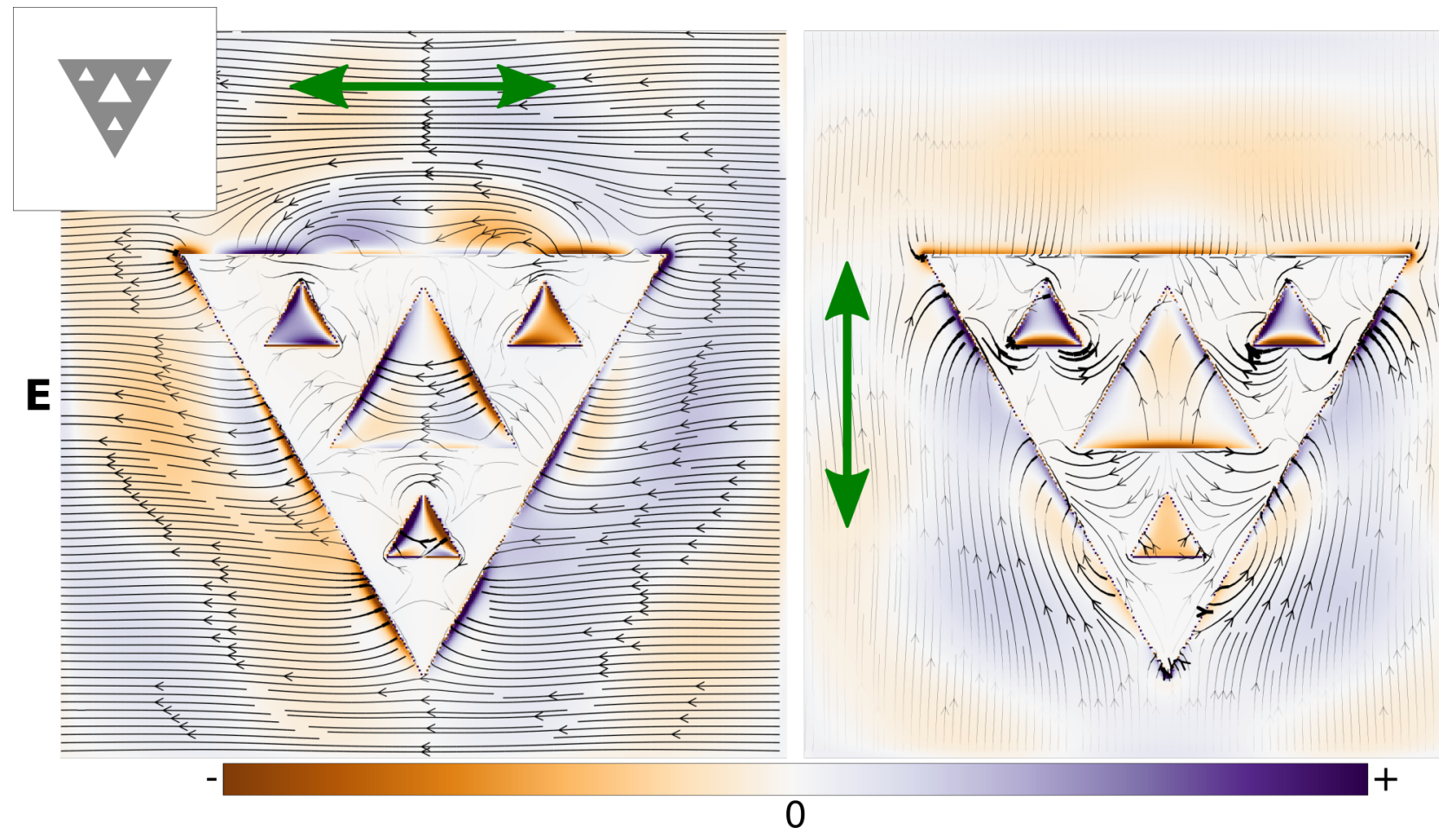

Figure S6: Simulated electric (E) fields for a plane just above the surface of the metal for a G2 fractal at $1.091 \mathrm{eV}(1136 \mathrm{~nm})$. 


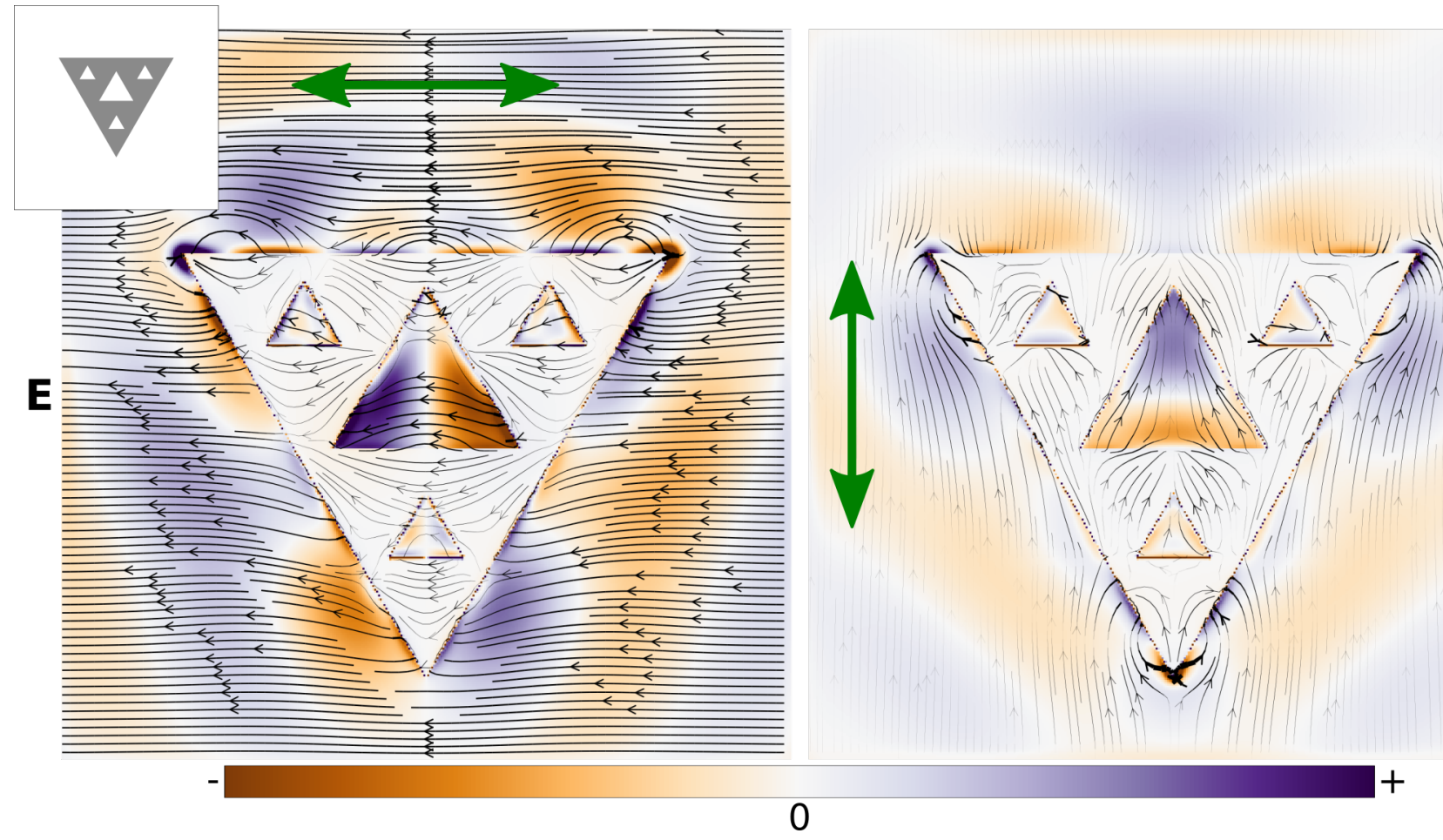

Figure S7: Simulated electric (E) fields for a plane just above the surface of the metal for a G2 fractal at $1.300 \mathrm{eV}(954 \mathrm{~nm})$.

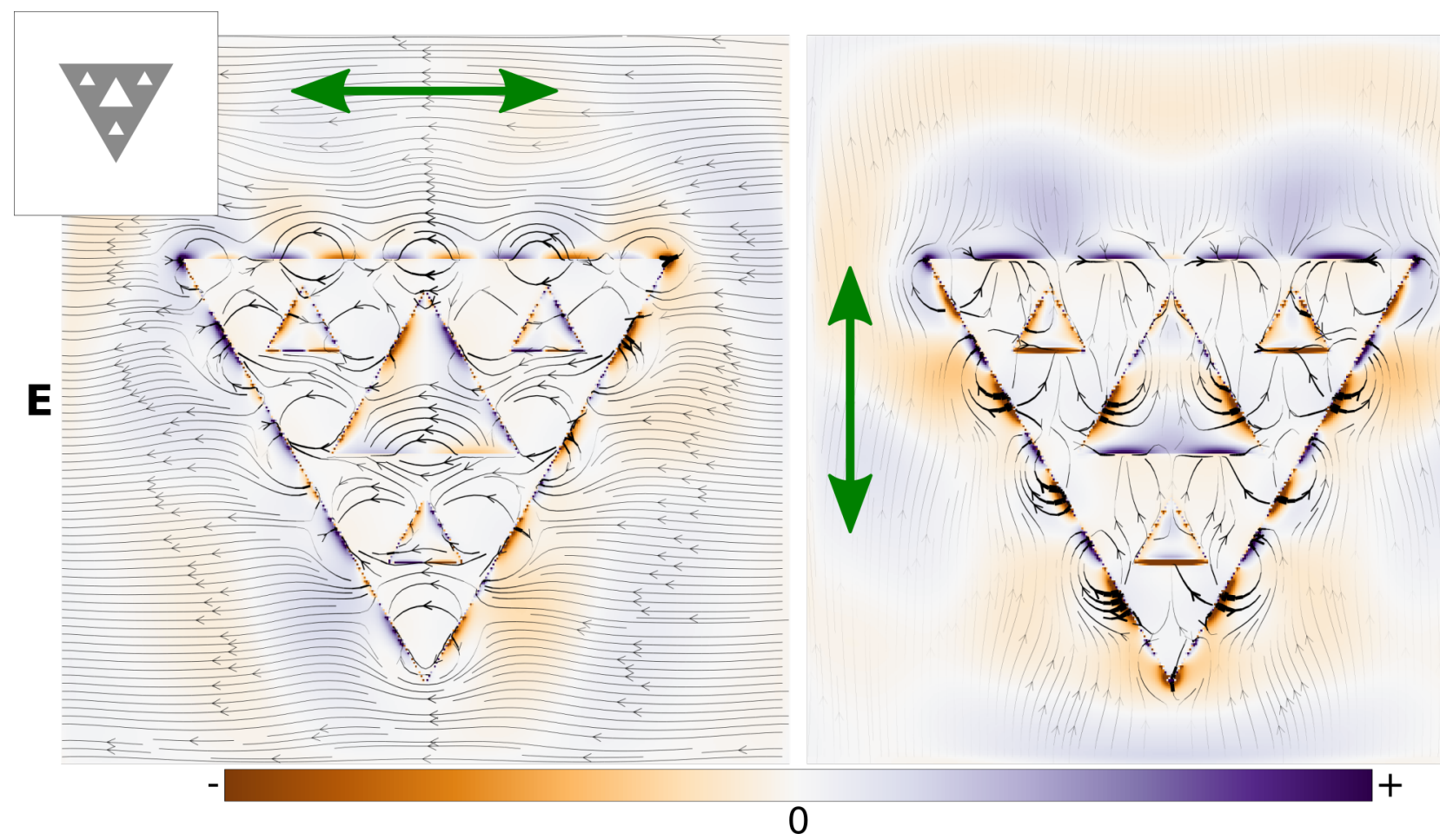

Figure S8: Simulated electric (E) fields for a plane just above the surface of the metal for a $\mathrm{G} 2$ fractal at $1.705 \mathrm{eV}(727 \mathrm{~nm})$. 


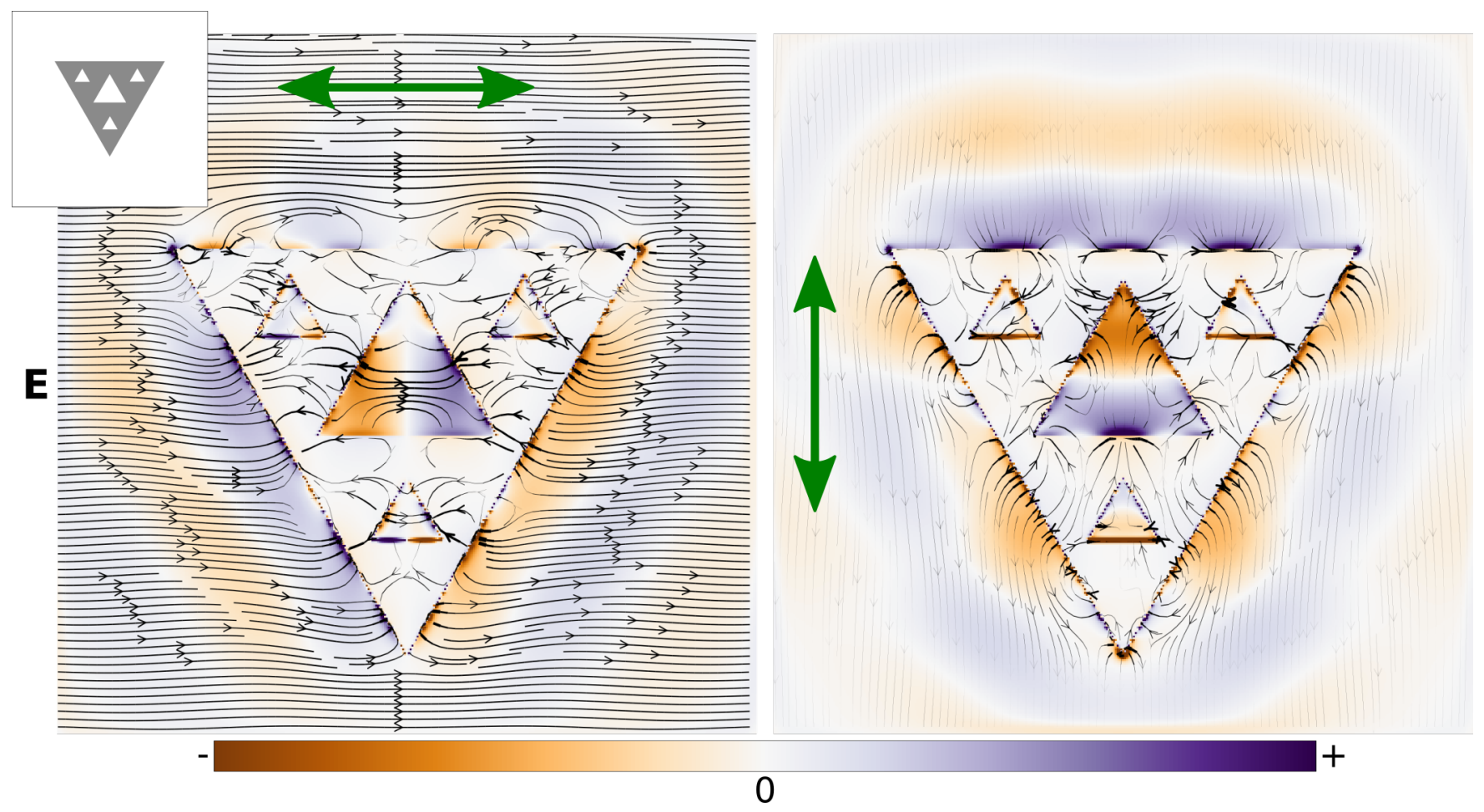

Figure S9: Simulated electric (E) fields for a plane just above the surface of the metal for a $\mathrm{G} 2$ fractal at $1.949 \mathrm{eV}(636 \mathrm{~nm})$. 


\section{References}

(1) Bicket, I. C.; Bellido, E. P.; McRae, D. M.; Lagugné-Labarthet, F.; Botton, G. A. Carving Plasmon Modes in Silver Sierpiński Fractals. ACS Photonics 2019, 6, 2974-2984.

(2) Bellido, E. P.; Manjavacas, A.; Zhang, Y.; Cao, Y.; Nordlander, P.; Botton, G. A. Electron Energy-Loss Spectroscopy of Multipolar Edge and Cavity Modes in Silver Nanosquares. ACS Photonics 2016, 3, 428-433.

(3) Campos, A.; Arbouet, A.; Martin, J.; Gérard, D.; Proust, J.; Plain, J.; Kociak, M. Plasmonic Breathing and Edge Modes in Aluminum Nanotriangles. ACS Photonics 2017, 4, 1257-1263. 